\title{
Expression of human FUS/TLS in yeast leads to protein aggregation and cytotoxicity, recapi- tulating key features of FUS proteinopathy
}

\author{
Kazuo Fushimi ${ }^{1}$, Charles Long ${ }^{1}$, Neha Jayaram ${ }^{1}$, Xiaoping Chen ${ }^{1}$, Liming Li $^{2}$, Jane Y. Wu ${ }^{1 凶}$ \\ ${ }^{1}$ Department of Neurology, Center for Genetic Medicine, Lurie Comprehensive Cancer Center, Northwestern University \\ Feinberg School of Medicine, $303 \mathrm{E}$. Superior, Chicago, IL 60611, USA \\ 2 Department of Molecular Pharmacology and Biological Chemistry, Northwestern University Feinberg School of Medicine, 303 \\ E. Superior, Chicago, IL 60611, USA \\ \ Correspondence: jane-wu@northwestern.edu \\ Received January 21, 2011 Accepted January 28, 2011
}

\section{ABSTRACT}

Mutations in the fused in sarcoma/translocated in liposarcoma (FUS/TLS) gene have been associated with amyotrophic lateral sclerosis (ALS). FUS-positive neuropathology is reported in a range of neurodegenerative diseases, including ALS and fronto-temporal lobar degeneration with ubiquitin-positive pathology (FTLDU). To examine protein aggregation and cytotoxicity, we expressed human FUS protein in yeast. Expression of either wild type or ALS-associated R524S or P525L mutant FUS in yeast cells led to formation of aggregates and cytotoxicity, with the two ALS mutants showing increased cytotoxicity. Therefore, yeast cells expressing human FUS protein recapitulate key features of FUSpositive neurodegenerative diseases. Interestingly, a significant fraction of FUS expressing yeast cells stained by propidium iodide were without detectable protein aggregates, suggesting that membrane impairment and cellular damage caused by FUS expression may occur before protein aggregates become microscopically detectable and that aggregate formation might protect cells from FUS-mediated cytotoxicity. The $\mathrm{N}$-terminus of FUS, containing the QGSY and G rich regions, is sufficient for the formation of aggregates but not cytotoxicity. The C-terminal domain, which contains a cluster of mutations, did not show aggregation or cytotoxicity. Similar to TDP-43 when expressed in yeast, FUS protein has the intrinsic property of forming aggregates in the absence of other human proteins. On the other hand, the aggregates formed by FUS are thioflavin T-positive and resistant to $0.5 \%$ sarkosyl, unlike TDP-43 when expressed in yeast cells. Furthermore, TDP-43 and FUS display distinct domain requirements in aggregate formation and cytotoxicity.

KEYWORDS FUS/TLS, protein aggregation, cytotoxicity

\section{INTRODUCTION}

Amyotrophic lateral sclerosis (ALS) and fronto-temporal lobar degeneration (FTLD) are devastating neurodegenerative disorders. There is no effective treatment for these ultimately fatal diseases. Recent discoveries of TDP-43 and FUS as characteristic components of neuronal inclusion bodies in FTLD have led to refinement of classification of FTLD, depending on immunohistochemistry of the tissue samples, as either FTLD-tau (tauopathy with tau-positive inclusions) or FTLD-U with tau-negative but ubiquitin-positive $(\mathrm{ub}+)$ neuronal inclusions (Urwin et al., 2010). Identification of mutations in the TDP-43 and FUS genes in ALS patients further highlights important roles of these DNA/RNA-binding proteins in the pathogenesis of ALS and FTLD-U (Lagier-Tourenne et al., 2010). Pathogenic mechanisms underlying these diseases remain to be elucidated.

FUS-positive inclusion bodies have been identified in sporadic FTLD, including atypical FTLD-U, neuronal intermediate filament inclusion disease, basophilic inclusion body disease and other neurodegenerative disorders such as 
Huntington disease (Munoz et al., 2009; Neumann et al., 2009; Woulfe et al., 2010). A large number of mutations in the FUS genes have been found in familial and sporadic ALS patients (Kwiatkowski et al., 2009; Vance et al., 2009; reviewed Lagier-Tourenne et al., 2010).

Originally identified as a fused protein produced by chromosome translocation in liposarcoma (Rabbitts et al., 1993), FUS is a DNA/RNA binding protein of the TAF15EWS-TLS (TET) protein family that includes the TATA-binding protein-associated factor (TAFII68/TAF15), the Ewing sarcoma (EWS) protein and TLS/FUS. The human FUS protein contains 526 amino acids and shares structural similarities with EWS and TAFII68/TAF15: an amino terminal region enriched in glutamine-glycine-serine-tyrosine (QGSY-rich domain), a glycine-rich region, a conserved RNA recognition motif (RRM), multiple arginine-glycine-glycine (RGG) repeats, and a C-terminal zinc finger motif. FUS is a multi-functional protein involved in many aspects of gene expression, including transcription, RNA processing, microRNA processing and translational regulation (reviewed in Buratti and Baralle, 2008; Lagier-Tourenne et al., 2010).

Yeast is a powerful model system for studying protein aggregate diseases. For example, expression of the Huntington exon-1 of variable polyQ-repeat lengths in yeast was shown to result in aggregate formation, with a positive correlation between the number of polyQ repeats and aggregate formation - the longer the repeats the higher the degree of aggregation (Krobitsch and Lindquist, 2000). Expression of the mammalian alpha synuclein had either no effect on growth with a plasma membrane localization or a severe growth inhibition with cytoplasmic inclusions, depending on its expression level (Outerio and Lindquist, 2003). Yeast models of mammalian prion protein (Tank et al., 2007), Alzheimer's disease associated A $\mathrm{A}-42$ (Bagriantsev et al., 2006), and ALS-linked TDP-43 (Johnson et al., 2008, 2009) have also been reported. To examine protein aggregation behavior in a simple eukaryotic system and to study the potential impact of human FUS protein expression on cell survival, we expressed the wild type and two ALS-associated mutant human FUS protein in yeast. Expression of full-length human FUS protein led to the formation of FUS protein aggregates and cytotoxicity. The $\mathrm{N}$-terminal QGSY and $\mathrm{G}$ rich fragments of FUS were sufficient to form aggregate by themselves, but did not cause cytotoxicity. The C-terminal fragment containing a zinc finger and glycine-rich domain did not cause cytotoxicity either. The aggregates were partially detergent resistant and FUS-expressing yeast showed positive staining by thioflavin T. Two ALS-associated mutations, R524S and P525L, showed increased cytotoxicity under certain conditions. Therefore, the key features of FUS proteinopathy, including cytotoxicity and protein aggregate formation, are recapitulated in this yeast model. Characterization of effects of human FUS expression in yeast reveals that FUS has features distinct from TDP-43.

\section{RESULTS}

\section{Expression of human FUS protein in yeast led to cytotoxicity and protein aggregate formation}

To examine the effects of FUS expression in yeast, we first expressed human FUS as a GFP-tagged protein in yeast under a GPD promoter in a $2 \mu$ plasmid that maintains a high copy number in yeast. Previously, it has been shown that a high expression level is critical for the detection of cytotoxicity caused by alpha synuclein expression (Outerio and Lindquist, 2003). If FUS is toxic to yeast, we reasoned that it would be more likely to be observed under a high-expression condition. Expression of either wild type FUS or ALS mutant (FUS ${ }^{R 524 S}$ and $\mathrm{FUS}^{\mathrm{P} 525 \mathrm{~L}}$ ) FUS showed a similar phenotype, with a significant reduction in cell growth and increased cytotoxicity (Fig. 1A). The toxicity was so severe that transformants expressing wild type or mutant FUS could not be re-streaked to form colonies (data not shown). To examine whether lowering the expression would reduce the toxicity, we expressed FUS under a TEF promoter in a plasmid carrying CEN, which maintains one copy per cell. As shown in Fig. 1A, the toxicity mediated by FUS was only slightly lowered, indicating FUS expression is extremely toxic to yeast under a constitutive expression condition. We then used an inducible Gal promoter to express FUS. Without induction of FUS expression, yeast grew well, similar to the control group. Upon galactose induction of FUS expression, however, there was a significant reduction in yeast growth or increase in toxicity, with the wild type and the two ALS mutant FUS, R524S and P525L showing similar effects (Fig. 1B).

Yeast cells expressing the wild type or ALS-mutant FUS under the Gal promoter were examined using fluorescent microscopy. Within $1 \mathrm{~h}$ after induction of FUS expression by galactose, FUS aggregates were detectable in the cytoplasm and nucleus, whereas GFP expressed in the control yeast distributed evenly in the cytosol (Fig. 2A). There was no obvious difference in aggregate formation between the wild type and ALS-mutants, R524S and P525L, when they were expressed at comparable levels as measured by Western blotting using a specific anti-FUS antibody (Fig. 2B). [RNQ $\left.{ }^{+}\right]$ prion can promote the de novo formation of other prions and enhance the aggregation of several glutamine/asparaginerich proteins (Derkatch et al., 2001). For example, the aggregation of GFP fusion proteins of poly-glutamineexpanded variant (Q68) of a fragment of the spinocerebellar ataxia type 3/Machado-Joseph Disease (MJD) or a glutamine-expanded Huntington disease protein (HttQ103) is dependent on the existence of $\left[\mathrm{RNQ}^{+}\right]$(Duennwald et al., 2006; Osherovich and Weissman, 2001). Similarly, aggregation of a GFP fusion of polyasparagine (polyN104) also requires the presence of $\left[\mathrm{RNQ}^{+}\right]$(Peters and Huang, 2007). We tested if the observed aggregation and cytotoxicity exhibited by FUS is $\left[\mathrm{RNQ}^{+}\right]$dependent. Interestingly, 

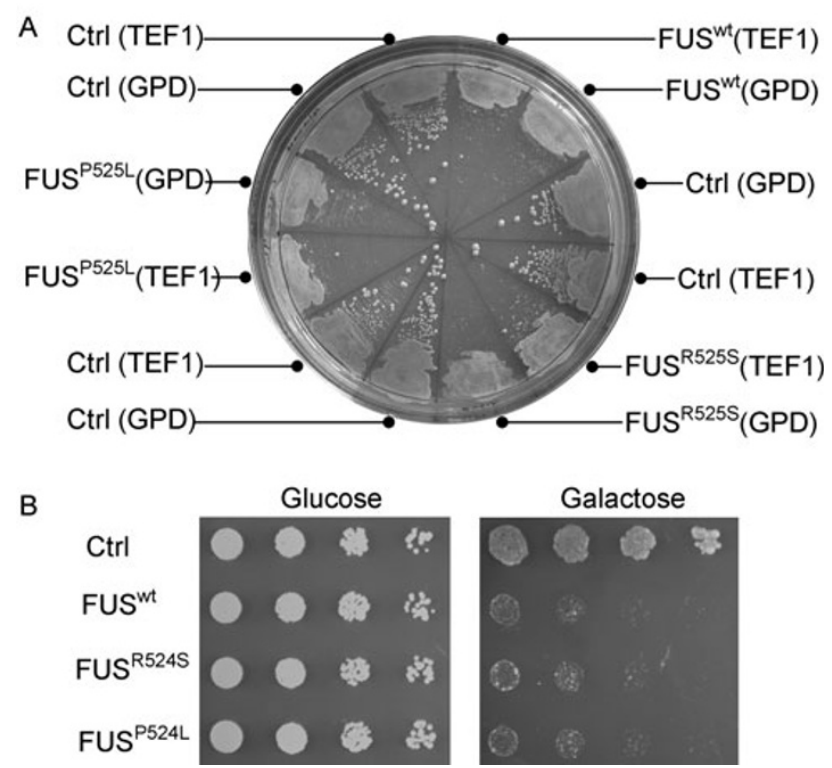

Figure 1. Expression of human FUS in yeast led to growth inhibition or cytotoxicity. (A) Yeast cells were transformed with either TEF1 or GPD vectors expressing vector control (Ctrl) or wild type (wt) or ALS-mutant (R524S, P525L) FUS. Transformation mixture was streaked on a $2 \%$ glucose SC-Ura plate and grown at $30^{\circ} \mathrm{C}$ for 3 days. Cells expressing wt or mutant FUS barely grew, whereas yeasts containing the vector control grew well. (B) Yeast cells transformed with a galactose-inducible FUS expression vector were grown in $2 \%$ raffinose liquid media and mid-logarithmic cultures were serially diluted before spotting onto glucose or galactose plates with images taken after 3 days.
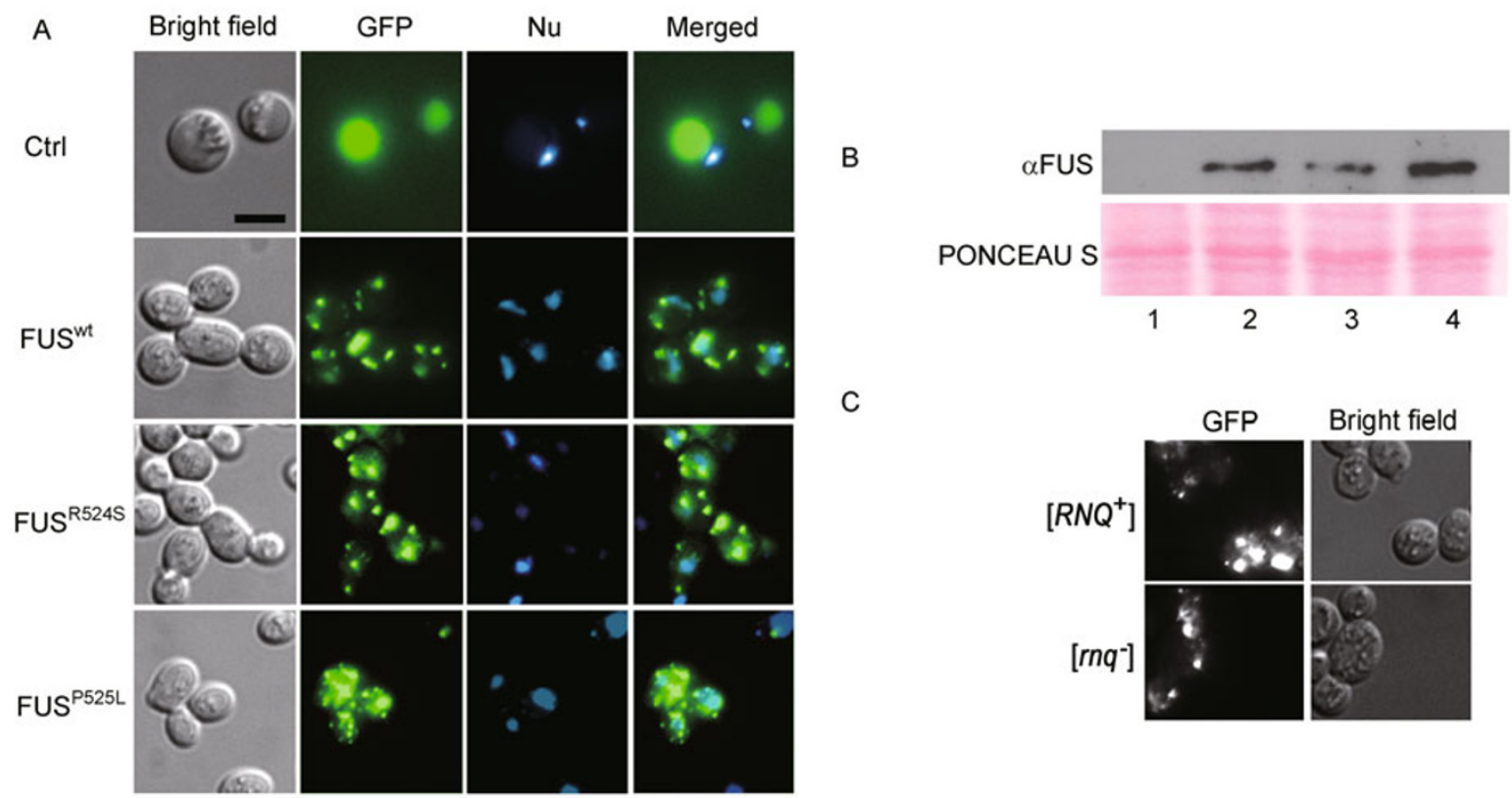

C

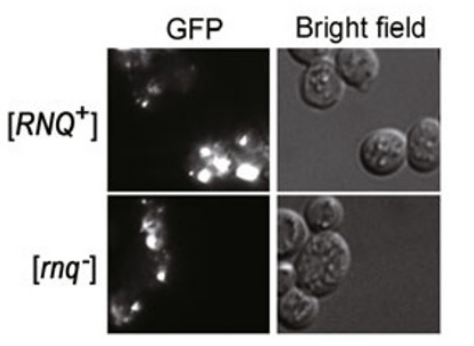

Figure 2. Expression of FUS in yeast led to the formation of protein aggregates. (A) Fluorescent microscopic images of FUSexpressing yeast with the galactose-inducible construct were induced by $2 \%$ galactose for $1 \mathrm{~h}$ before imaging $(\times 100$ magnification). FUS-GFP or control GFP expression is shown in green, and nuclei stained with Hoechst 33342 in blue. The scale bar indicates $5 \mu \mathrm{m}$. (B) Western blot with anti-FUS antibody showing equivalent levels of expression among the Wt FUS and ALS-mutant, R524S and P525L (lanes 2-4). Lane 1 contains the control GFP expressing yeast. (C) The formation of FUS aggregates is independent of [RNQ ${ }^{+}$. 
we found that FUS aggregated similarly in isogenic $\left[R N Q^{+}\right]$ and $\left[r n q^{-}\right]$cells, causing similar growth inhibition and cytotoxicity in both cell types (data not shown), unlike HttQ103 and polyN104 whose aggregation requires the presence of $\left[R N Q^{+}\right]$. Thus, FUS aggregation is $\left[R N Q^{+}\right]$ independent.

To examine whether FUS expression caused cytotoxicity, we carried out a staining assay using propidium iodide (PI), a nucleic acid-binding and membrane-impermeable dye that is excluded from healthy cells. More than $20 \%$ of cells expressing FUS were positive for PI staining when FUS expression was induced either at room temperature or at $30^{\circ} \mathrm{C}$ (Fig. $3 \mathrm{~A}$ and $3 \mathrm{~B}$ ). This indicates that expression of human FUS protein in yeast does not simply cause growth arrest but leads to cell death. Quantification of PI-positive cells showed that cell death in FUS expressing cells was significantly higher than the control GFP-expressing cells and the difference between wild type FUS and P525L mutant was statistically significant at both room temperature and $30^{\circ} \mathrm{C}$ (Fig. $3 \mathrm{C}$ and $3 \mathrm{D}$ ). At $30^{\circ} \mathrm{C}$, the level of cell death in R524S mutant was significantly higher than that detected in the yeast expressing the wild type FUS protein (Fig. 3D). These results suggest that ALS-associated mutations, R524S and P525L, may increase cytotoxicity associated with FUS expression. It was noticed that a significant fraction of FUS expressing cells were $\mathrm{PI}$-positive before microscopic protein aggregates were detectable (marked by arrowheads, Fig. 3A, 3B and 3E).

\section{Expression of $\mathrm{N}$-terminal domain was sufficient for aggregate formation but not for cytotoxicity}

To determine the domains responsible for aggregate formation and cytotoxicity, we constructed a series of truncation mutants of FUS. N370 contained QGSY rich, $G$ rich and RRM sequences. N285 contained QGSY and G rich sequences, and C392 contained RGG, zinc finger and NLS sequences at
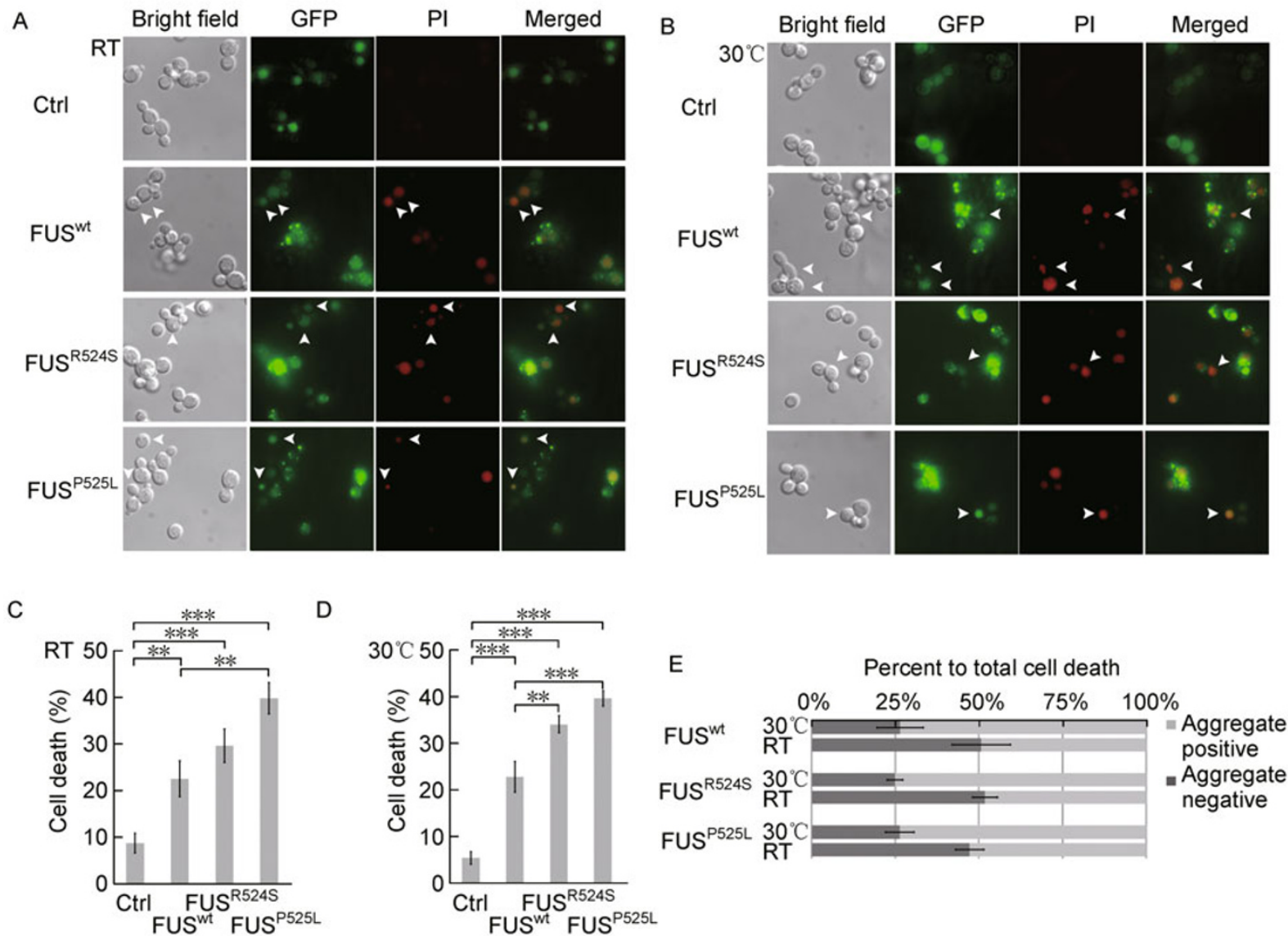

Figure 3. Propidium iodide (PI) staining revealed that FUS expression is cytotoxic. (A and $B$ ) FUS expression was induced by $2 \%$ galactose in the presence of $\mathrm{PI}$ for $3 \mathrm{~h}$ at either room temperature (RT) (A) or $30^{\circ} \mathrm{C}(\mathrm{B})$. GFP and PI signals were detected by fluorescent microscopy. (C and D) Quantification of PI-positive cells among yeast cells with or without aggregates at RT $(C)$ or $30^{\circ} \mathrm{C}$ (D). In control cells expressing GFP only, fewer than $5 \%$ of the cells were PI-positive. Cell death in mutant FUS expressing yeast cells was significantly higher as compared with the control yeast expressing GFP or the wild type FUS $\left(^{* *}: p<0.01\right.$; ${ }^{* * *}: p<0.001$; Student's T test). Four microscopic fields were observed, and a total of more than 200 cells were scored for each group. (E) Quantification of PIstained cells containing or lacking aggregates. 
the $\mathrm{C}$ terminus (Fig. 4A). Expression of N370 and N280 but not $\mathrm{C} 392$ in yeast resulted in formation of aggregates (Fig. 4B). We also introduced R524S and P525L mutations into the C392 fragment and examined if these mutations at the $C$ terminus led to protein aggregation. These ALSmutants expressed in the carboxyl terminal fragment containing amino acids 392-526 did not result in aggregate formation in yeast cells (Fig. 4C). As compared with the full length wild type FUS (FL-FUS), none of these truncation mutants showed cytotoxicity (Fig. 4D) suggesting that FUS cytotoxicity may require the zinc finger domain and/or RGG domain in addition to the RRM and N-terminal domains.

\section{FUS protein aggregates were sarkosyl-resistant and thioflavin T-positive}

We examined detergent solubility of FUS aggregates in yeasts. Yeast cell homogenates were treated with $0.5 \%$ sarkosyl, and then centrifuged to obtain sarkosyl insoluble fractions. The insoluble fractions were analyzed by Western blotting (Fig. 5A; lanes 9-12). The majority of FUS was detected in the insoluble fraction, whereas GFP protein produced in the vector control yeast was detected only in the sarkosyl soluble fraction (Fig. 5B). These results show FUS aggregates in yeast cells are resistant to $0.5 \%$ sarkosyl.

NLS

A

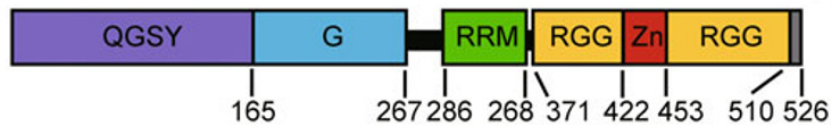

FL

N370

N285

C392

B

Ctrl
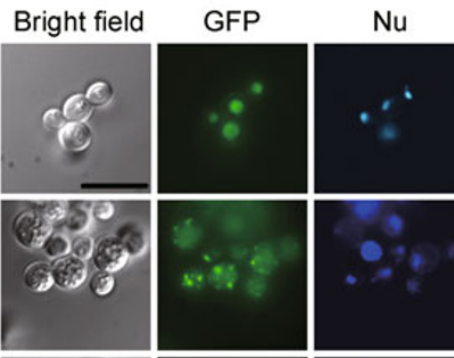

Merged

FL-FUS

N370
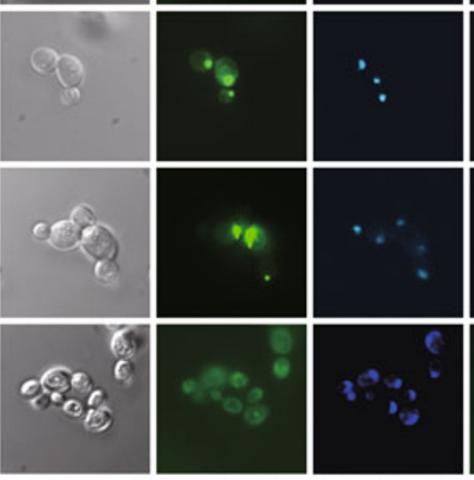

$2 \%$ galactose with Gall promote

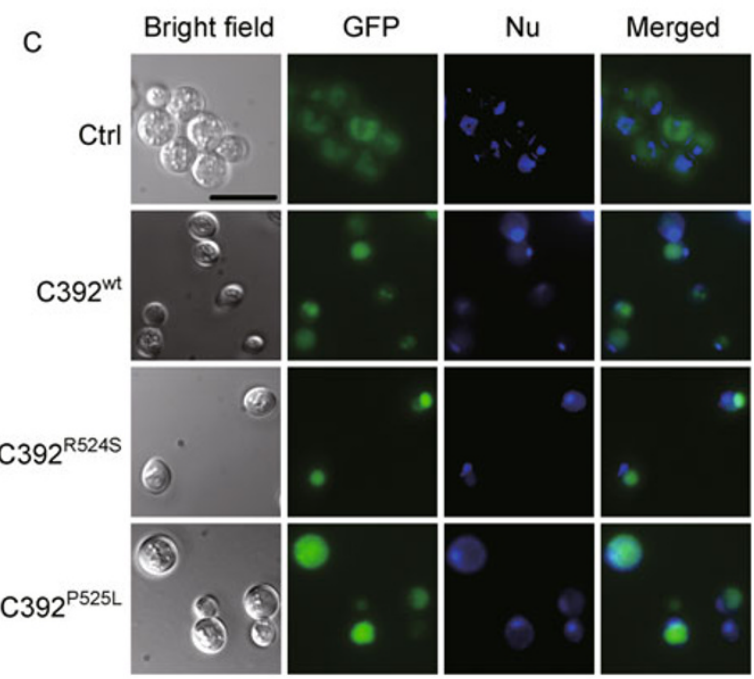

GPD promoter

D

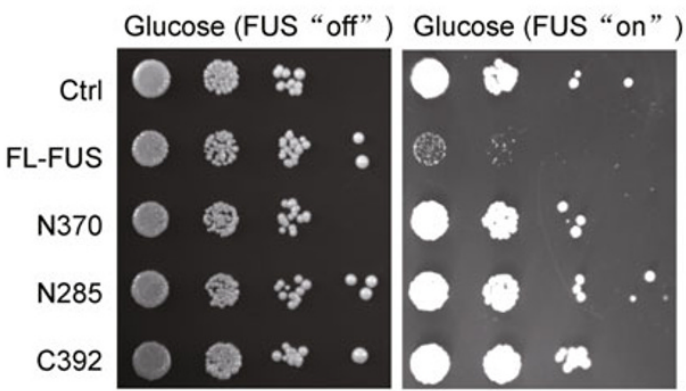

Figure 4. Deletion analyses revealed FUS protein domain was involved in aggregate formation. (A) A schematic diagram of FUS. QGSY, GIn/Gly/Ser/Tyr-rich sequence; G, Gly-rich sequence; RRM, RNA recognition motif; RGG, Arg-Gly-Gly repeat; Zn, Zinc finger; NLS, nuclear localization signal; FL, full-length; N370, truncated FUS (amino acids 1 to 370); N285, truncated FUS (amino acids 1-285); C392, truncated FUS (amino acids 392-526). (B) FL or mutant FUS was expressed with the galactose inducible construct following induction with $2 \%$ galactose. GFP signal is shown in green; nuclei, blue. The scale bar indicates $10 \mu \mathrm{m}$. (C) Wild type and mutant $C$ terminal fragments of FUS were expressed as GFP fusion proteins, not forming aggregates in yeast. The scale bar indicates $10 \mu \mathrm{m}$. (D) The full-length FUS but not truncation mutants caused cytotoxicity. 


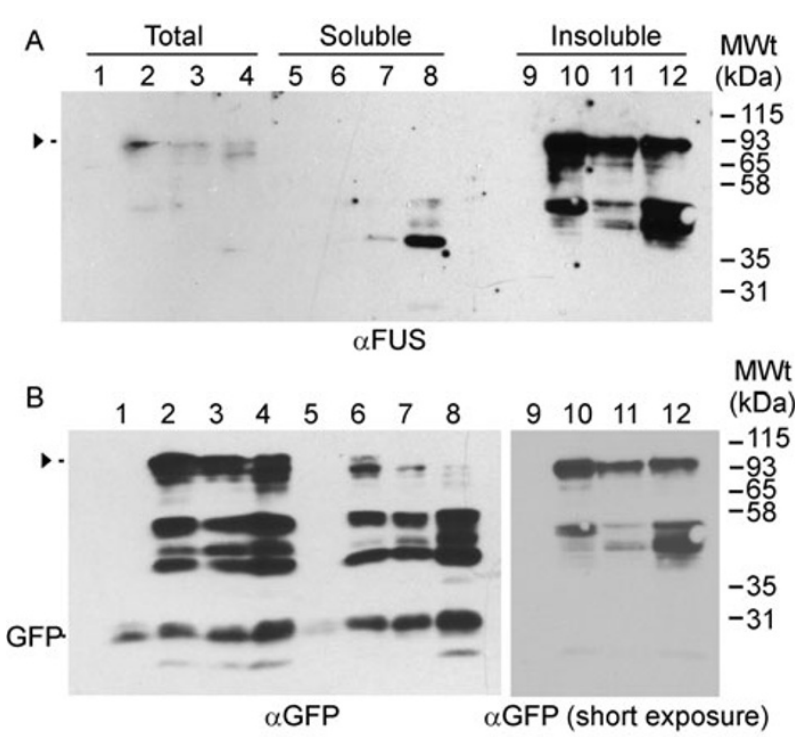

Figure 5. FUS aggregates in yeast were insoluble in $\mathbf{0 . 5 \%}$ Sarkosyl. Sarkosyl insoluble fractions were obtained and examined as described in MATERIALS AND METHODS. Each fraction was analyzed by Western blot using anti-FUS or anti-GFP antibody. FUS existed in insoluble fractions predominantly (panel A). (A and B) Lanes 1, 5 and 9 contained GFP; lanes 2, 6 and 10: wild type FUS; lanes 3, 7 and 11: R524S; lanes 4, 8 and 12: P525L.

When FUS expression was induced for a shorter period of time in the presence of galactose and the yeast lysates were prepared in the presence of $2 \%$ sarkosyl, most proteins were detected in soluble fractions (Fig. S1). These results show FUS aggregates in yeast cells are resistant to $0.5 \%$ sarkosyl.

To examine whether FUS protein aggregates in yeast may exist in amyloid form, we carried out thioflavin T staining. Yeast cells expressing control RFP showed no thioflavin T signal (Fig. 6). However, yeast cells expressing FUS exhibited strong thioflavin T staining signals (Fig. 6). This suggests that FUS expression in yeast may have amyloid-like features.

\section{DISCUSSION}

FUS is one of the major components of inclusions in tissues of patients affected by non-SOD type ALS and atypical FTLD-U, especially those with ubiquitin-positive, basophilic and NissI positive inclusions (Munoz et al., 2009; Neumann et al., 2009). However, the role of FUS in aggregate formation and in the diseases remains unclear. We examined FUS protein in yeast, which has been extensively used for characterization of yeast prions and aggregation-prone proteins associated with neurodegeneration. When expressed in yeast, FUS protein caused significant cytotoxicity and formed aggregates (Fig. 2 and 3). Cytotoxicity was increased by two

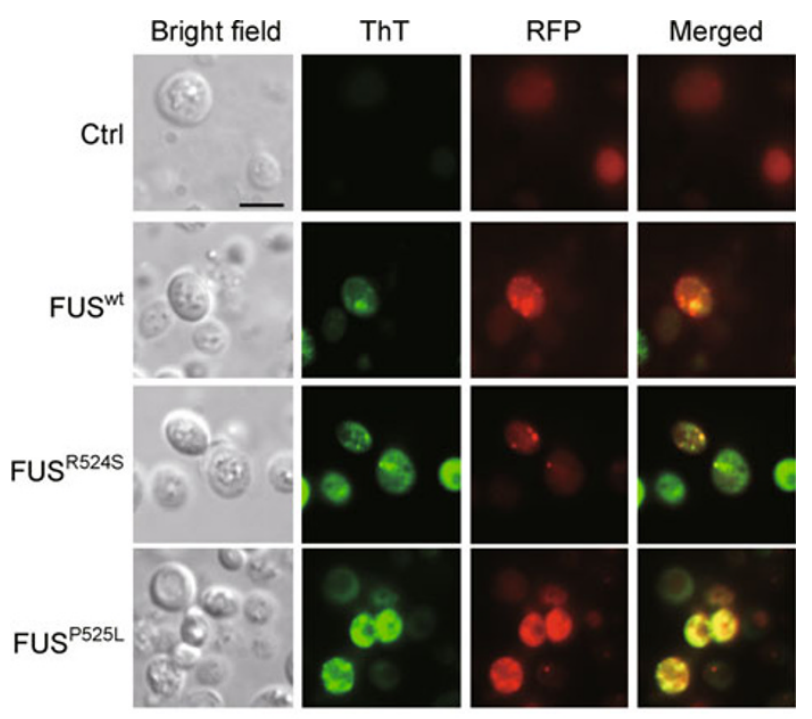

Figure 6. FUS aggregates in yeast were thioflavin T positive. RFP tagged FUS was expressed in yeast. Thioflavin $T$ staining was carried out as described in MATERIALS AND METHODS. ThT signals are shown in green; RFP, in red. The scale bar indicates $5 \mu \mathrm{m}$.

ALS-associated mutations, R524S and P525L, as compared with yeast expressing the wild type FUS (Fig. 3), although expression of either Wt or mutant FUS led to the formation of aggregates. This is consistent with the observations that FUS-positive inclusion bodies are detected in FTLD-FUS patients without detectable FUS mutations (Munoz et al., 2009; Neumann et al., 2009).

Protein aggregations in neurodegenerative disorders have been studied extensively in yeast cells (Outeiro and Giorgini, 2006). For example, $Q$ extended huntingtin forms detergent insoluble aggregates in both yeast and human cells (Krobitsch and Lindquist, 2000; Outeiro and Giorgini, 2006). TDP43 also forms aggregates and shows cytotoxicity (Johnson et al., 2008, 2009). Some yeast proteins are known to have prion-like properties in yeast, and a bioinformatics approach using those proteins predicts a number of proteins showing an aggregation property in yeast (Alberti et al., 2009).

TDP-43 distribution is shifted into a sarkosyl insoluble fraction in FTLD-TDP patients (Neumann et al., 2006). In a fly model of FTLD-TDP, the sarkosyl insoluble fraction contains TDP-43 and the aggregates are not dissociated even by $2 \%$ SDS in SDS-PAGE analysis (Li et al., 2010). We observed that FUS aggregates in yeasts were resistant to $0.5 \%$ sarkosyl but not to $2 \%$ sarkosyl (Fig. 5 and Fig. S1). Thus the solubility properties of FUS protein seem to be distinct from those of TDP-43.

Interestingly, thioflavin T staining was detected when FUS was expressed in yeast cells, suggesting beta-sheet like structure may exist in the FUS protein aggregates (Fig. 6). Beta-sheet stacking by misfolding is believed to play an 
important role in the formation of amyloid fibrils in vivo and in vitro. Amyloid fibrils are often detectable by thioflavin $\mathrm{T}$. Consistent with our ThT staining data, fibrils have been detected by electron microscopy in FUS-positive inclusions from juvenile ALS patients (Huang et al., 2010). It should be noted that ThT-positive FUS-containing inclusion bodies have not been reported in tissue samples.

It remains possible that FUS causes cell death by both loss-of-function and gain-of-function cytotoxicity. In mammalian cells FUS shuttles between the nucleus and the cytoplasm (Zinszner et al., 1997). ALS-associated mutations could disrupt the function of nuclear localization signals (Dormann et al., 2010; Gal et al., 2010; Ito et al., 2010). Consequently, mutant FUS accumulates in the cytosol. Under the stressed conditions, mutant FUS forms stress granules; however, wild type FUS stays in the nuclei (Bosco et al., 2010; Dormann et al., 2010; Gal et al., 2010). This could explain why many FUS mutations in ALS are autosomal dominant except for the H517Q mutation, which was reported to inherit in an autosomal recessive manner (Kwiatkowski et al., 2009). Growth inhibition and aggregate formation are observed when TDP-43 is expressed in yeast (Johnson et al., 2008). Together with this study, these results suggest that aggregate formation and cytotoxicity may represent common features among neurodegenerative disorders in which FUS and/or TDP-43 positive inclusions have been observed, such as ALS, FTLD, Alzheimer's disease (AD) and Parkinson's disease (PD).

The N-terminal region in human FUS protein consisting of the QGSY and G rich sequences has been predicted to have prion-like features (Cushman et al., 2010). Consistent with this prediction, our experiments show that the N285 domain was sufficient for the aggregate formation but did not cause cytotoxicity (Fig. 4B). N285 does not contain the RRM. Expanding the sequence to include the RRM, the truncation mutant N370 still did not cause cytotoxicity. This is different from findings in yeast expressing human TDP-43, in which addition of either one or two RRMs to the carboxyl terminus was sufficient to cause both aggregation and cytotoxicity (Johnson et al., 2008). The cytotoxicity and aggregate formation properties of both FUS and TDP-43 are separable in yeast cells. In the case of FUS, the addition of the RRM to the aggregate forming $\mathrm{N}$-terminal domain was not sufficient to cause cytotoxicity. In addition, the carboxyl terminal glycinerich fragment of FUS did not have either aggregate forming or cytotoxic activities. Together with published studies (Johnson et al., 2008, 2009), our data show that FUS and TDP-43 have distinct domain requirements of their aggregate formation and cytotoxicity in yeast cells.

In this paper, we show that expression of human FUS in yeast causes cytotoxicity and formation of aggregates, recapitulating key features of FUS-positive neurodegenerative diseases. The amino-terminal domain of FUS, including the QGSY and G rich sequences, is sufficient to form aggregates, but is unable to induce the cytotoxic effects of FUS. The FUS expressing yeasts are thioflavin T-positive, suggesting the accumulation of intermolecular beta-sheet stacks. Yeast cells expressing human FUS protein should be useful for studying aggregate formation and cytotoxic properties of human FUS protein and helpful in investigating the molecular pathogenesis of FUS-positive neurodegenerative disorders including ALS and FTLD.

\section{MATERIALS AND METHODS}

\section{Yeast expression vectors and yeast strains}

The open reading frames corresponding to wild type FUS or ALSmutants: R524S and P525L, were inserted into yeasts expression vectors, p426-GPD, p416-TEF or p426-Gall, in frame with EGFP or RFP tag at the carboxyl-termini of FUS. A series of FUS truncation mutants were prepared. The sequences of all constructs were confirmed by DNA sequencing.

The yeast strain used in most experiments was 74D-694(MATa his3 leu2 ura3) $\left[\mathrm{RNQ}^{+}\right]$. No difference in protein aggregation or toxicity was observed when 74D-694 (MATa his3 leu2 ura3) [RNQ] was used. Yeast cultures were grown in rich media (YPD) or synthetic media containing either $2 \%$ glucose, raffinose or galactose at different concentrations as specified.

\section{Yeast culture, transformation, spotting assay and yeast cytotoxicity assay}

Yeast transformation and culture procedures were carried out according to standard protocols. Spotting assay was performed using serially diluted mid-log phase culture using a Frogger (V \& $P$ Scientific) onto synthetic solid media containing glucose or galactose. Yeast was cultured on plates at $30^{\circ} \mathrm{C}$ for 3 days.

To grow yeast transformants of FUS expression vectors, synthetic media lacking uracil and containing raffinose for the Gal 1 promoter or glucose for constitutive promoters was used. To induce FUS expression by the Gal1 promoter, $0.1 \%-2 \%$ of galactose was used.

\section{PI staining}

PI was added to liquid yeast culture $(5 \mu \mathrm{g} / \mathrm{mL})$ for $3 \mathrm{~h}$ at either room temperature or $30^{\circ} \mathrm{C}$ followed by fluorescent microscopy.

\section{Fractionation and detergent solubility assay}

The control or FUS expressing yeast cells were harvested by centrifugation at $3000 \mathrm{~g}$ for $10 \mathrm{~min}$ and treated with zymolyase 20T $(1 \mathrm{mg} / \mathrm{mL})$ in $20 \mathrm{mM}$ Tris- $\mathrm{HCl}(\mathrm{pH} 7.4), 1.2 \mathrm{M}$ sorbitol, and $10 \mathrm{mM} 2-$ mercaptoethanol for $30 \mathrm{~min}$ at $30^{\circ} \mathrm{C}$. Cells were collected by centrifugation at $1000 \mathrm{~g}$ for $10 \mathrm{~min}$ and resuspended with $20 \mathrm{mM}$

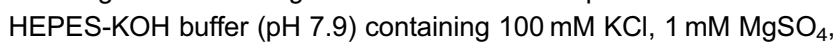
$0.2 \mathrm{mM} \mathrm{CaCl}_{2}, 20 \%$ glycerol $5 \mathrm{mM}$ AEBSF and Complete Protease Inhibitor Cocktail (Roche Applied Science). Proteins were extracted by vortex with glass beads and cell debris was removed by centrifugation at $800 \mathrm{~g}$ for $5 \mathrm{~min}$. Protein concentration was measured by the Bradford protein assay and adjusted at $1 \mathrm{mg} / \mathrm{mL}$. Sarkosyl was 
added into the lysates to final concentrations of $0.5 \%$ and $2 \%$. The mixture was incubated at room temperature for $5 \mathrm{~min}$ and then insoluble fractions were collected by centrifugation at $16,000 \mathrm{~g}$ for $1 \mathrm{~h}$ at $4^{\circ} \mathrm{C}$. Pellets were washed with lysis buffer once and centrifuged at $16,000 \mathrm{~g}$ for $30 \mathrm{~min}$ to eliminate residual protein in soluble fractions. The pellet was recovered with $5 \mu \mathrm{L}$ SDS sample buffer and boiled for $2 \mathrm{~min}$, and then $5 \mu \mathrm{L}$ of $10 \mathrm{M}$ urea was added to load onto SDS PAGE gel.

\section{Fluorescent microscopy}

A Zeiss Axioplan microscope was used to detect fluorescent signals from EGFP, RFP, Hoechst 33342, and Thioflavin T with YFP, RFP, UV, and CFP filter sets, respectively. Thioflavin staining was carried out as described before (Johnson et al., 2008).

\section{ACKNOWLEDGEMENTS}

We thank members of the Wu lab for stimulating discussions and helpful suggestions. We thank David Zhang for technical assistance in the early stage of the work. JYW is supported by NIH and James S. McDonnell Foundation. LL is supported by NIH (R01NS056086).

\section{ABBREVIATIONS}

ALS, amyotrophic lateral sclerosis; FTLD, fronto-temporal lobar degeneration; FTLD-U, fronto-temporal lobar degeneration with ubiquitin-positive pathology; PI, propidium iodide; RGG, arginineglycine-glycine; RRM, RNA recognition motif

\section{REFERENCES}

Alberti, S., Halfmann, R., King, O., Kapila, A., and Lindquist, S. (2009). A systematic survey identifies prions and illuminates sequence features of prionogenic proteins. Cell 137, 146-158.

Bagriantsev, S.N., Kushnirov, V.V., and Liebman, S.W. (2006). Analysis of amyloid aggregates using agarose gel electrophoresis. Methods Enzymol 412, 33-48.

Bosco, D.A., Lemay, N., Ko, H.K., Zhou, H., Burke, C., Kwiatkowski, T. J. Jr, Sapp, P., McKenna-Yasek, D., Brown, R.H. Jr, and Hayward, L.J. (2010). Mutant FUS proteins that cause amyotrophic lateral sclerosis incorporate into stress granules. Hum Mol Genet 19, 4160-4175.

Buratti, E., and Baralle, F.E. (2008). Multiple roles of TDP-43 in gene expression, splicing regulation, and human disease. Front Biosci 13, 867-878.

Cushman, M., Johnson, B.S., King, O.D., Gitler, A.D., and Shorter, J. (2010). Prion-like disorders: blurring the divide between transmissibility and infectivity. J Cell Sci 123, 1191-1201.

Derkatch, I.L., Bradley, M.E., Hong, J.Y., Liebman, S.W., (2001). Prions affect the appearance of other prions: the story of [PIN(+)]. Cell 106, 171-182.

Dormann, D., Rodde, R., Edbauer, D., Bentmann, E., Fischer, I., Hruscha, A., Than, M.E., Mackenzie, I.R., Capell, A., Schmid, B., et al. (2010). ALS-associated fused in sarcoma (FUS) mutations disrupt Transportin-mediated nuclear import. EMBO J 29, 2841-2857.

Duennwald, M.L., Jagadish, S., Giorgini, F., Muchowski, P.J., and
Lindquist, S. (2006). A network of protein interactions determines polyglutamine toxicity. Proc Natl Acad Sci U S A 103, 11051-11056.

Gal, J., Zhang, J., Kwinter, D.M., Zhai, J., Jia, H., Jia, J., and Zhu, H. (2010). Nuclear localization sequence of FUS and induction of stress granules by ALS mutants. Neurobiol Aging. July 29. [Epub ahead of print]

Huang, E.J., Zhang, J., Geser, F., Trojanowski, J.Q., Strober, J.B., Dickson, D.W., Brown, R.H. Jr, Shapiro, B.E., and Lomen-Hoerth, C. (2010). Extensive FUS-Immunoreactive Pathology in Juvenile Amyotrophic Lateral Sclerosis with Basophilic Inclusions. Brain Pathol 20, 1069-1076

Ito, D., Seki, M., Tsunoda, Y., Uchiyama, H., and Suzuki, N. (2010). Nuclear transport impairment of amyotrophic lateral sclerosislinked mutations in FUS/TLS. Ann Neurol 8, 11.

Johnson, B.S., McCaffery, J.M., Lindquist, S., and Gitler, A.D. (2008). A yeast TDP-43 proteinopathy model: Exploring the molecular determinants of TDP-43 aggregation and cellular toxicity. Proc Natl Acad Sci U S A 105, 6439-6444.

Johnson, B.S., Snead, D., Lee, J.J., McCaffery, J.M., Shorter, J., and Gitler, A.D. (2009). TDP-43 is intrinsically aggregation-prone, and amyotrophic lateral sclerosis-linked mutations accelerate aggregation and increase toxicity. J Biol Chem 284, 20329-20339.

Krobitsch, S., and Lindquist, S. (2000). Aggregation of huntingtin in yeast varies with the length of the polyglutamine expansion and the expression of chaperone proteins. Proc Natl Acad Sci U S A 97, 1589-1594.

Kwiatkowski, T.J., Jr., Bosco, D.A., Leclerc, A.L., Tamrazian, E., Vanderburg, C.R., Russ, C., Davis, A., Gilchrist, J., Kasarskis, E.J., Munsat, T., et al. (2009). Mutations in the FUS/TLS gene on chromosome 16 cause familial amyotrophic lateral sclerosis. Science 323, 1205-1208.

Lagier-Tourenne, C., Polymenidou, M., and Cleveland, D.W. (2010). TDP-43 and FUS/TLS: emerging roles in RNA processing and neurodegeneration. Hum Mol Genet 19, R46-R64.

Li, Y., Ray, P., Rao, E.J., Shi, C., Guo, W., Chen, X., Woodruff, E.A. 3rd, Fushimi, K., and Wu, J.Y. (2010). A Drosophila model for TDP43 proteinopathy. Proc Natl Acad Sci U S A 107, 3169-3174.

Munoz, D.G., Neumann, M., Kusaka, H., Yokota, O., Ishihara, K., Terada, S., Kuroda, S., and Mackenzie, I.R. (2009). FUS pathology in basophilic inclusion body disease. Acta Neuropathol 118, 617-627.

Neumann, M., Rademakers, R., Roeber, S., Baker, M., Kretzschmar, H.A., and Mackenzie, I.R. (2009). A new subtype of frontotemporal lobar degeneration with FUS pathology. Brain 132, 2922-2931.

Neumann M, Sampathu DM, Kwong LK, Truax AC, Micsenyi MC et al. (2006) Ubiquitinated TDP-43 in frontotemporal lobar degeneration and amyotrophic lateral sclerosis. Science 314, 130-133.

Osherovich, L.Z., and Weissman, J.S. (2001). Multiple GIn/Asn-rich prion domains confer susceptibility to induction of the yeast [PSI(+)] prion. Cell 106(2), 183-194.

Outeiro, T.F., and Lindquist, S. (2003). Yeast cells provide insight into alpha-synuclein biology and pathobiology. Science 302, 1772 -1775 .

Peters, T.W., and Huang, M. (2007). Protein aggregation and polyasparagine-mediated cellular toxicity in Saccharomyces cerevisiae. Prion 1(2), 144-153.

Rabbitts, T.H., Forster, A., Larson, R., and Nathan, P. (1993). Fusion 
of the dominant negative transcription regulator $\mathrm{CHOP}$ with a novel gene FUS by translocation $\mathrm{t}(12 ; 16)$ in malignant liposarcoma. Nat Genet 4, 175-180.

Tank, E.M., Harris, D.A., Desai, A.A., and True, H.L. (2007). Prion protein repeat expansion results in increased aggregation and reveals phenotypic variability. Mol Cell Biol 27, 5445-5455.

Urwin, H., Josephs, K.A., Rohrer, J.D., Mackenzie, I.R., Neumann, M., Authier, A., Seelaar, H., Van Swieten, J.C., Brown, J.M., Johannsen, P., et al., and the FReJA Consortium. (2010). FUS pathology defines the majority of tau- and TDP-43-negative frontotemporal lobar degeneration. Acta Neuropathol 120, 33-41.
Vance, C., Rogelj, B., Hortobágyi, T., De Vos, K.J., Nishimura, A.L., Sreedharan, J., Hu, X., Smith, B., Ruddy, D., Wright, P., et al. (2009). Mutations in FUS, an RNA processing protein, cause familial amyotrophic lateral sclerosis type 6. Science 323, 1208-1211.

Woulfe, J., Gray, D.A., and Mackenzie, I.R. (2010). FUSimmunoreactive intranuclear inclusions in neurodegenerative disease. Brain Pathol 20, 589-597.

Zinszner, H., Sok, J., Immanuel, D., Yin, Y., and Ron, D. (1997). TLS (FUS) binds RNA in vivo and engages in nucleo-cytoplasmic shuttling. J Cell Sci 110, 1741-1750. 